Cahiers d'études italiennes

\title{
L'odore del sangue di Goffredo Parise: fascinazione della gioventù, tentazioni di un reporter
}

Matteo Giancotti

\section{CpenEdition}

\section{Journals}

Edizione digitale

URL: http://journals.openedition.org/cei/833

DOI: $10.4000 /$ cei.833

ISSN: 2260-779X

\section{Editore}

UGA Éditions/Université Grenoble Alpes

\section{Edizione cartacea}

Data di pubblicazione: 15 septembre 2006

Paginazione: 207-217

ISBN: 978-2-84310-086-4

ISSN: 1770-9571

Notizia bibliografica digitale

Matteo Giancotti, «L'odore del sangue di Goffredo Parise: fascinazione della gioventù, tentazioni di un reporter», Cahiers d'études italiennes [Online], 5 | 2006, online dal 15 mars 2008, consultato il 26 mars 2021. URL: http://journals.openedition.org/cei/833 ; DOI: https://doi.org/10.4000/cei.833 


\title{
L'ODORE DEL SANGUE DI GOFFREDO PARISE
}

\author{
FASCINAZIONE DELLA GIOVENTÙ, TENTAZIONI DI UN REPORTER*
}

\author{
Matteo Giancotti \\ Università di Padova
}

1. Uno dei pochi margini di discussione lasciati da Cesare Garboli nella sua esauriente Prefazione a L'odore del sangue, ${ }^{1}$ il romanzo postumo di Goffredo Parise, riguarda l'interpretazione di un tratto fondamentale dell'opera. Il ragazzo di cui Silvia s'innamora tradendo il marito Filippo, il giovane violento e senza volto che incarna in breve l'ossessione di Filippo, è fascista. Costituzionalmente fascista, si direbbe, dal momento che questa connotazione politica precede nel romanzo ogni sua descrizione fisica, morale, "culturale". Alle domande del marito che la interroga per la prima volta intorno a questo altro uomo della sua vita, Silvia risponde infatti, sminuendo:

"Ma niente" [...] "è un ragazzo, figurati, un ragazzo che ho conosciuto per strada.

Fascista per di più. Figurati, alla mia tenera età". (p. 11; nostro corsivo)

Dato fondamentale, dunque, quello dell'appartenenza politica del giovane, accampato agli albori della vicenda e più volte ossessivamente ribadito nel corso del libro. Come lo interpreta Garboli? Come una funzione razionalizzante, un tentativo di disinnescare la devastante portata simbo-

\footnotetext{
* Una redazione più ampia di questo scritto è stata anticipata, in accordo con gli organizzatori del convegno, su "studi novecenteschi", XXXII, 69, gennaio-giugno 2005.

1. Parise G., L'odore del sangue, a cura di C. Garboli e G. Magrini, Milano, Rizzoli, 1997 (da qui si cita con l'indicazione dei numeri arabi per le pagine del romanzo e dei numeri romani per la Prefazione di Garboli e la Nota al testo di Magrini). Scritto nel 1979, sigillato e mai più riletto fino a due mesi dalla morte, il romanzo non fu mai chiaramente approvato o rifiutato dall'autore (secondo la testimonianza di Garboli, p. VI, Parise disse soltanto "che alcune parti del romanzo erano buone, altre da rifare").
} 
lica del fallo del ragazzo che ha ammaliato Silvia. ${ }^{2}$ In realtà la connotazione fascista non ha l'effetto di contenere il dolore ma di esasperarlo, rendendolo più terribile e minaccioso, più irrazionale.

Silvia ama un truce e violento ragazzo. Tanto violento e prepotente quanto interiormente fragile e qualunquista. Ignorante. Afasico. Epilettico. Con i segni sulle braccia di un tentato suicidio. Che cosa poteva renderlo più minaccioso e mostruoso? L'appartenenza neofascista, che ha punti di contatto solo apparenti con la retorica celebrazione della virilità da parte del fascismo storico o mussoliniano, già demolita con vivacissima ironia dal Parise de Il prete bello. Per capire meglio l'aria minacciosa e irrazionale che spira dalla gioventù neofascista bisogna rituffarsi negli anni Settanta, "a Roma, nell'odore del sangue” (p. 229). Partendo dal romanzo, dall'importante rappresentazione di una Roma putrefatta e violenta che prende forma agli occhi di Filippo durante una passeggiata notturna:

Erano non so più se le tre o le quattro, e Roma mostrava il suo volto notturno fatto sostanzialmente di spazzatura vagante, di qualche pantera della polizia, urlante, di ragazzi in giubbotti di cuoio che sfrecciavano rombando in motocicletta. Eccoli, erano loro, i giustizieri della notte, quelli che avevano assassinato Pasolini, quelli che avevano stuprato le ragazze del Circeo, quelli che avevano bruciato un somalo dormiente su un letto di cartoni, "per scherzo". Intravedevo le loro facce, anche nella velocità della corsa. Parevano facce americane, alcune bionde e butterate, altre nere dai capelli ricci, di arabi americanizzati. Erano, nella loro anonima e meccanica criminalità, le facce di Roma. Dei figli della borghesia o delle borgate di Roma, la stessa cosa: entrambi vestiti allo stesso modo, entrambi con fattezze di tipo americano e criminoide con appena una punta di quella vanità e brutalità mediterranea e romana che si vede appunto a Roma. (pp. 90-91)

La descrizione di questi ragazzi combacia con le sembianze che Filippo immagina, e poi verifica nella realtà, appartenere al ragazzo di Silvia. ${ }^{3}$ Non è ancora guarita dunque in Parise, all'epoca della stesura del romanzo, la cicatrice lasciata dall'uccisione brutale di Pasolini. È una ferita aperta che ancora agisce nella sua immaginazione, che lo perseguita, ${ }^{4}$ che trova per la

2. "[...] il Narratore è impegnato a contenere la sofferenza, a misurarla, a circoscrivere la prepotenza del fallo antagonista ragionandoci sopra, cercando di abbatterlo con argomenti intellettuali, dandogli una connotazione fascista, [...]”. (p. XX)

3. "Un ragazzo qualunque del generone romano [...] certamente nevrotico come mille altri, dei Parioli, di Piazza del Popolo, del Pantheon, ma anche delle borgate, del sottoproletariato, uno delle centinaia di migliaia di ragazzi di cui è impossibile riconoscere l'origine sociale. [Frutto] di quel mutamento, di quella omologazione antropologica di cui parlava Pasolini”. (pp. 229-230)

4. "Pianse come nessun altro sui suoi amici, sulle mani di Comisso morente, su una fotografia incollata sul loculo dei resti di Pasolini, [...]"; così Naldini N. Con Goffredo Parise. Atti del convegno (Treviso 19 settembre 1987), a cura di N. Naldini, Treviso, Zoppelli, 1988, p. 40. 
prima volta uno sfogo di scrittura in questo romanzo. Avvicinare il giovane fascista amato da Silvia alla schiera dei linciatori romani, dei giustizieri per noia e per scherzo, dei fantasmi dell'omicidio Pasolini (un'enorme massa di gente dagli identici connotati fisionomici e sociali), ha l'effetto di incupire e illividire la vicenda del romanzo. Il neofascismo del giovane amante è un'ombra ambigua e terrifica che infetta ulteriormente, anziché razionalizzare, la tragedia sessuale e sentimentale di Silvia e Filippo; un'ombra reale, esistente. Una piaga nell'Italia degli anni Settanta e di Roma in particolare.

Nei due anni precedenti alla sua morte, Pasolini aveva trattato spesso, in interventi pubblici durissimi e dolorosi, il tema del neofascismo, spesso mettendolo in relazione al radicale mutamento di costumi provocato nei giovani italiani dall'affermazione della civiltà dei consumi. Non esiste più - diceva - quella Roma popolare che egli amava e immaginava antropomorfizzata nelle "sembianze di un tipico ragazzo romano di borgata: cioè bruno, olivastro, con l'occhio nero, il corpo aitante. [...] Snello, non precisamente atletico. Un po' come gli arabi che non sono atletici ma sono, diciamo così, fatti armoniosamente"; la civiltà dei consumi e dei mass-media ha trasformato Roma in "una delle tante piccole città italiane. Piccolo-borghesi, meschine, cattoliche, impastate di inautenticità e di nevrosi". 5 Inasprendo il proprio giudizio sui nuovi giovani italiani ("questi giovani d'oggi che la pagheranno cara e che mi fan pena"), Pasolini dichiarava: "sono odiosi. I ragazzi dai quindici ai venti ai ventuno ai ventidue anni sono odiosi. Poveretti. Non sanno più parlare, sono completamente afasici, sono dei presuntuosi. Sono proprio una povera cosa. [...] hanno perso la loro individualità, son tutti uguali, fascisti, antifascisti, studenti, operai, borghesi, sottoproletari, delinquenti. Se tu vai per strada in una borgata romana o nelle periferie milanesi e cammini e vedi dei gruppi di giovani, niente, ma proprio niente ti può far dire guardandoli se quelli sono o no dei delinquenti e se fra cinque minuti ti ammazzeranno. Io lo trovo semplicemente spaventoso". ${ }^{6}$ Un passo da L'odore del sangue riguardante ancora il degrado sociale di Roma (chiaramente cassato da Parise e riprodotto dai curatori in appendice con il titolo Roma) dimostra, nelle evidenti affinità con queste ultime affermazioni di Pasolini, l'origine pasoliniana di molte angosciose atmosfere del romanzo postumo di Parise. ${ }^{7}$

5. Pasolini P.P., Quant'eri bella Roma, in Id., Saggi sulla politica e sulla società, a cura di W. Siti e S. De Laude, Milano, Mondadori, 1999, pp. 1701-1704.

6. Id., Eros e cultura, Saggi, op. cit., p. 1715.

7. “[...] Girellai per Piazza del Popolo. La piazza, così bella in altri tempi, era disseminata di 
Il ragazzo di Silvia esce esattamente da queste inquietanti analisi, integrate dall'esame "autoptico" che Parise, grande osservatore della realtà, conduce sul campo trovandosi perfettamente d'accordo, come dice in un passo sopra riportato del romanzo, con le posizioni di Pasolini. ${ }^{8}$ Non per razionalizzare la potenza sessuale del giovane, dunque, Parise gli attribuisce i connotati politici del neofascismo, ma al contrario per rendere più esasperante la carica minacciosa di questo individuo senza volto, per rendere più teso "l'odore di agguato" che Filippo percepisce nella casa coniugale profanata dalle visite di questo picchiatore "per scherzo". Fallisce infatti il suo tentativo di esorcizzare con la ragione il tradimento della moglie spaventosamente in balia del fallo sempre eretto di un giovane (quel fallo che, monstrum naturale incomprensibile alla ragione, continua in fondo ad ipnotizzare per la sua potenza sia la moglie che il marito). Né gli riesce quell'esercizio di sociologia che dovrebbe portarlo, attraverso la scomposizione analitica del carattere, della provenienza sociale e degli obiettivi politici del ragazzo, a liberarsi dall'ossessione della gelosia. La ragione non vale nei confronti di questi giovani nuovi, di questa "nuova razza" di uomini che sono fascisti senza un perché, che sono fascisti come potrebbero essere antifascisti, che uccidono per scherzo, per puro arbitrio; che in un delirio di ignoranza, prepotenza, nevrosi e vanità infantile teorizzano "il culto della forza fisica, del disprezzo delle donne" e "il disimpegno assoluto, la teorizzazione del non far nulla, di non agire" (così Silvia definisce il giovane e i suoi amici, aggiungendo anche, brutalmente: " $\mathrm{Ci}$ sono loro e il loro cazzo e basta”, pp. 175-176). La minuziosa ricomposizione delle tessere sociali e "culturali" che dovrebbero portare Filippo alla

rifiuti e seduti, anzi stravaccati, sulle sedie esterne del Bar Rosati, che mi ricordava sempre il mio primo incontro con Silvia, stavano dei ragazzi. Anche loro in blue-jeans, in maglietta, con un giubbotto nero, i capelli ricci e arabi tagliati corti. Alcuni andavano, altri venivano con grosse motociclette. [...] Così doveva essere, esattamente così, come migliaia in tutta Roma, il ragazzo di Silvia. [...] Mi avvicinai per guardarli. [...] Anch'essi, di tanto in tanto, mi gettavano un'occhiata, ma non era l'ora, quella, del linciaggio. Il linciaggio era già stato forse compiuto da qualche parte durante la notte. La spedizione punitiva di quelle facce aveva itinerari casuali che nascevano sempre come scherzi, come occasioni di fare qualche risata e soprattutto di esprimere se stessi. Il pestaggio di un omosessuale, per esempio, se non, puro e semplice, l'assassinio e la fuga, come topi nelle fogne" (pp. 231-233).

8. Sul difficile rapporto tra Pasolini e Parise cfr. Marco Belpoliti, Settanta, Torino, Einaudi, 2001: "dopo la tragica morte di Pasolini, Parise sul "Corriere" sembra fare propri i temi e le questioni del suo "avversario". [...] È come se perdendo il suo "antagonista" - a tratti detestato, a tratti amato, ma sempre ammirato - Parise avesse assunto su di sé anche la sua parte" (p. 82).

9. Così Perrella S. Fino a Salgareda. La scrittura nomade di Goffredo Parise, Milano, Rizzoli, 2003, p. 117. Ma cfr. anche Pasolini, La prima, vera rivoluzione di destra, in Scritti corsari e ora Saggi, op. cit., p. 289: "Ci stanno sostituendo degli uomini nuovi, portatori di valori tanto indecifrabili quanto incompatibili con quelli, così drammaticamente contraddittori, finora vissuti”. 
comprensione della psicologia del giovane, e quindi a dare un volto razionalmente rappresentabile al tradimento di Silvia (il che basterebbe, secondo il razionalista Filippo, a superare il trauma e a vincere la gelo$\operatorname{sia}^{10}$ ), non centra il bersaglio: questo ragazzo continua a presentarsi con volto di sfinge. ${ }^{11}$ Ed è una sfinge terrifica, abbiamo detto, densa di un'aura potenzialmente e inspiegabilmente omicida, casuale e perciò non prevedibile nelle sue intenzioni. A ciò è da aggiungere il ricordo raccapricciante della morte di Pasolini, che affiora in Parise come crux desperationis, delitto giuridicamente irrisolto ma in realtà addirittura previsto dalla vittima che lungamente aveva studiato e osservato, addirittura guardato in faccia, quelli che sarebbero stati - secondo Parise - i suoi carnefici. Niente di più irrazionale e inquietante dunque, nel neofascismo degli anni Settanta, secondo l'interpretazione di Pasolini adottata in blocco da Parise (che in quegli anni, era straordinariamente recettivo rispetto a molte delle questioni sollevate dall'intellettuale friulano ${ }^{12}$ ): "Tutti sapevamo, nella nostra coscienza, che quando uno di quei giovani decideva di essere fascista, ciò era puramente casuale, non era che un gesto, immotivato e irrazionale"; ${ }^{13}$ e ancora: "Il nuovo fascismo [...] è un tumore centrale [...] è un po' la media delle aspirazioni nevrotiche di una società i cui elementi parossistici, i conformisti esemplari, sono gli estremisti che mettono le bombe e i killers. [...] Io ritengo che nulla è stato intrapreso contro l'irrazionalismo e la disperazione di una certa categoria di giovani $[\ldots]$ ". ${ }^{14}$

10. Cfr. p. 69: "Partivo dall'idea che conoscere le cose significa esorcizzarle".

11. Effigie immobile più che personaggio capace di azione e pensiero, il ragazzo è figura caratterizzata da emblemi che segnalano la sua appartenenza alla nuova società dei consumi prima che a un'organizzazione politica: "Sta sempre con quel maledetto giubbotto addosso, non se lo leva mai" dice di lui Silvia (p. 45). E Filippo ne integra l'identikit: "Portava naturalmente blue-jeans e scarpe da ginnastica. Una catena d'oro al collo" (p. 53). Infine, emblema degli emblemi, compare la Coca Cola: "Fascista, che fa l'alba tutte le notti, che non ha la più lontana idea di lavorare e va in palestra e beve Coca Cola" - dice Filippo -. "Come fai a saperlo?" "L'ho visto in frigidaire, ne hai una scorta". "Sì, beve solo Coca Cola e non fuma", disse Silvia [...]" (p. 101).

12. Cfr. un articolo uscito il 30 giugno 1974 sul "Corriere della Sera" (Il rimedio è la povertà): "I giovani "comprano" ideologia al mercato degli stracci ideologici così come comprano blue jeans al mercato degli stracci sociologici (cioè per obbligo, per dittatura sociale). [...] Ha ragione Pasolini quando parla di nuovo fascismo senza storia" (Parise G., Verba volant. Profezie civili di un anticonformista, a cura di S. Perrella, Firenze, Liberal, 1998, p. 78).

13. Pasolini P.P., Il vero fascismo e quindi il vero antifascismo in Scritti corsari; ora in Saggi, op. cit., p. 317.

14. Id., Da un fascismo all'altro, in Id., Il sogno del centauro, a cura di J. Duflot, Roma, Editori Riuniti, 1983, ora in id., Saggi, op. cit., pp. 1530-1531. 
2. Silvia è soggiogata dal prepotente odore della vita e della gioventù che il sesso del suo ragazzo venticinquenne emana. Filippo lo sa, lo percepisce anche dalle piccole tracce che il linguaggio di Silvia dissemina durante i loro infiniti dialoghi. Sono i cosiddetti lapsus. Filippo è uno psicanalista, non può fare a meno di notarli, e di interpretarli. ${ }^{15}$ Una situazione del genere sarebbe, per qualsiasi marito innamorato della propria moglie (Filippo dice di esserlo), orrenda: la realizzazione di un incubo, probabilmente il peggiore degli incubi maschili. Per di più, il ragazzo che tiene soggiogata Silvia appartiene ad Ordine nuovo, "una pericolosa società mezza segreta di teppisti fascisti romani” (p. 52), il cui solo nome basta a proiettare nella mente di Filippo "un suono sinistro" che emana "odore di sangue marcio, di assassinio, di stupro, di bravate delinquenziali” (pp. 101-102).

Silvia non è solo una donna che tradisce il marito; è una donna in pericolo, che rischia la vita, e Filippo ne è consapevole:

Ero preso dal terrore. Non soltanto di perdere Silvia per sempre, ma di perdere Silvia per sempre e in un modo oscuro, sinistro, in cui sentivo fino a provarne il vomito l'odore nauseabondo del sangue. (p. 121)

Bisogna agire per salvarla, anche con la forza se necessario, come Filippo prospetta a se stesso: sottrarla "al plagio di questo stronzo di ragazzo” (p. 135). L'altro modo di salvarla, gli spiega Mario, amico e collega psicanalista cui Filippo si rivolge in preda alla disperazione, è quello di lasciare che questa storia impossibile si esaurisca da sé, come è destinata a fare per ragioni sociali e anagrafiche, e che Silvia ritorni da sola e, per così dire naturalmente, al marito. Filippo, secondo Mario, dovrebbe solo essere comprensivo e starle vicino, non abbandonarla. Soprattutto, non esercitare la psicanalisi su di lei, perché "potrebbe essere pericoloso e lo sai bene" (p. 134).

Filippo ha dunque due possibilità di azione per salvare Silvia. Una che potremmo definire massimalista, che prevede l'uso della forza, e una moderata che impone pazienza, affetto, comprensione, sospensione del giudizio e dell'analisi. Non adotterà nessuna delle due.

15. "Molto prepotente, nel nuovo linguaggio reticente di Silvia, significava che questo ragazzo aveva su di lei, come si dice, pieni poteri. Che lei era alla sua mercé; anzi, per essere più precisi e brutali, alla mercé del suo cazzo" (p. 88). 
L'ipotesi massimalista, quella dell'azione romantica, dell'opposizione eroica al destino, è bruciata in partenza. Primo perché Filippo è un intellettuale e questo tipo di azione diretta, di intervento immediato nella vita, non gli è possibile se non debolmente e quasi unicamente sul piano del pensiero. ${ }^{16}$ Secondo perché nel romanzo italiano, e Parise lo sapeva bene essendo Moravia uno dei suoi maestri, la possibilità di azione romantica, lo sdegno e l'ira di fronte all'oltraggio e al tradimento, era già stata liquidata come "arcaica" fin dal tempo de Gli indifferenti (1929). Impossibile, inapplicabile, defunta, come prova sulla propria pelle il giovane Michele, protagonista del romanzo d'esordio di Moravia: incapace di reagire con forza di fronte a Leo còlto in un tentativo malriuscito di seduzione della "sua" donna (Lisa); incapace di una autentica e vitale reazione di orgoglio di fronte allo stesso Leo che, pur essendo l'amante di sua madre, ha sedotto e rovinato anche la sorella Carla, oltraggiandone il pudore. ${ }^{17}$ Edoardo Sanguineti ha parlato di questi aspetti nei termini di una "impossibilità della tragedia", impossibilità-impotenza dell'azione romantica e grande, incapacità di liberare la violenza come naturale conseguenza dell'istinto borghese ferito. ${ }^{18}$ "Michele figura dell'impotenza", scrive Sanguineti a proposito del protagonista degli Indifferenti. ${ }^{19}$ Anche riguardo a Filippo dell'Odore del sangue si è parlato esattamente di "impotenza” ad agire. ${ }^{20} \mathrm{Ma}$ se negli Indifferenti l'impotenza di Michele origina

16. Cfr. p. 73: "Devo agire perché Silvia non sia innamorata di qualcun altro, chiunque sia, devo fare qualcosa per non perderla. E architettavo infinite azioni per così dire belliche affinché la cosa potesse avvenire; al tempo stesso, proprio nel momento dell'azione, ecco la [narcosi], l'anestesia, l'impossibilità di agire. E una volta subentrata l'anestesia che corrispondeva a una certa relativa calma della mente e dei nervi, come avviene appunto durante la pausa di una battaglia, ragionavo". Reale, ma abortito, il tentativo di strangolare la moglie in un albergo di Venezia: "Mi prese una furia tremenda [...] e in quello stesso istante mi alzai sul letto e l'afferrai al collo. Silvia ebbe un leggero sorriso, tra stupito e scherzoso, perché spesso, per scherzo, le facevo un gesto simile. Ma io non scherzavo ed ero deciso. Strinsi forte il collo finché divenne paonazza. A quel punto mi resi conto di quanto stavo facendo e alzai i pugni stretti, mugolando e come preso dalla pazzia. La guardai, lo ricordo molto bene, con un odio tremendo e omicida. Silvia aveva preso paura" (p. 213).

17. Questi i pensieri di Michele: "Bisogna dire qualcosa di più forte... io sono il fratello oltraggiato dall'amante di sua madre nell'onore di sua sorella (tutte queste parole virtuose e familiari gli facevano un ridicolo effetto come se fossero state arcaiche): bisogna trovare qualche cosa di più duro... magari è necessario esagerare..." Ma tra queste ironiche falsità la sua triste stanchezza aumentava: avrebbe voluto lasciare questa commedia [...]" (Moravia A., Gli indifferenti, introduzione di Sanguineti E., Milano, Bompiani, 1997, p. 238; corsivo nostro).

18. Sanguineti E., Introduzione a Moravia A., Gli indifferenti, op. cit., pp. XVI-XX.

19. Ibid., p. XXI.

20. Sulla scorta di esplicite ammissioni del narratore: "Mi sentivo ed ero impotente, come nella realtà dei fatti. Come non potevo in nessun modo agire, così non potevo in nessun modo fare all'amore" (p. 226). L'impotenza d'azione è strettamente correlata all'impotenza sessuale di cui il 
"l'impossibilità della tragedia" (che rivela il costituzionale gretto pragmatismo di una borghesia sempre capace di ricomporre le rovine con un gioco al ribasso delle idealità), l'impotenza ad agire di Filippo è nell'Odore del sangue il vero motore della tragedia: qui nessuno dei protagonisti, pur essendo in fondo tutti borghesi, ragiona e agisce alla luce dell'utilitarismo borghese che ha come norma imperante il "salvare il salvabile", l'economia della vita residua. ${ }^{21}$ Qui ogni esperienza è vissuta fino all'estrema conseguenza, senza troppa considerazione del rischio della vita, senza paura dello scardinamento dell'istituto familiare. L'odore del sangue mette in scena una tragedia moderna, un "gioco al massacro" (p. 36) in cui ogni azione è libera e incondizionata dalla morale: un funambolismo senza reti. $\mathrm{Si}$ misurano in queste divergenze il tempo passato dai romanzi di Moravia, le nuove possibilità di azione e reazione che uomo e donna possono esercitare all'interno - e all'esterno - dell'istituto della coppia.

Anche i consigli dell'amico-collega Mario, di cui dicevamo poc'anzi, sono per Filippo inaccettabili. Filippo, alter ego di Parise (o Parise tout court come propone Perrella ${ }^{22}$ ), non può, come Silvio, protagonista de L'amore coniugale di Moravia, entrare nella "parte" di marito della donna adultera, aspettare che la bufera passi e che il tempo gli riconsegni la moglie, magari provata, magari invecchiata di dieci anni, ma viva. Filippo deve capire, interrogare, arrivare alla verità e al nucleo delle ragioni che hanno generato la paurosa sbandata di Silvia. Deve farlo ad ogni costo: "Io devo sapere $[. .$.$] devo sapere la verità, solo sapendola sarò in grado di fare$ qualcosa per lei al momento in cui sarà necessario" (p. 135). Deve quindi, ciò che in effetti fa, sospendere qualsiasi tipo di azione e ragionare, ragionare e interrogare Silvia, tirarle fuori tutta la verità. Questa - dice a se stesso, mentendo - è l'unica terapia per sopravvivere al dolore e per salvare Silvia: quando le cose saranno dette con chiarezza, non faranno più paura e sarà facile esorcizzarle. In realtà, questa specie di maieutica ossessiva, esercitata su Silvia con infiniti e dolorosi dialoghi, non è nient'altro che

cinquantacinquenne Filippo comincia ad avvertire il fantasma: $c f r$. Brunetta M., Lutto, sensualità mentale, erotismo senile: "L'odore del sangue" di Goffredo Parise, in "Esperienze letterarie", XXVI, 2001, n. 4, p. 67.

21. Spiega infatti Filippo: "In casi normali, quella che ho definita una signora borghese romana come Silvia, quando vede il pericolo vero, se ne fugge via a gambe levate. Ma tutto mi diceva che questo non era un caso normale" (p. 177); e ancora: "l'innocenza di Silvia nell'accettare gente così, non fosse altro che per la sua schizzinoseria e il suo temperamento tremante, farla venire in casa, sedere alla sua tavola, preparare lei stessa il cibo, mi pareva un'altra spia di un tipo di plagio che andava oltre la novità, oltre la cotta per un ragazzo, oltre i limiti, appunto, che la borghesia impone a ogni pazzia a cui ha voglia di abbandonarsi” (pp. 178-179).

22. Perrella S., Fino a Salgarèda, op. cit., p. 115. 
una maieutica della distruzione. Non perché Filippo voglia con questo suo metodo - come pure ammette varie volte nel corso della narrazione uccidere lui stesso Silvia, farsi "mandante" del suo assassinio (p. 230): questo è soltanto un espediente narrativo. Piuttosto perché dietro a Filippo (in Filippo) c'è Parise, lo scrittore entomologo, l'attentissimo giornalista osservatore della realtà umana in tutte le sue forme. È lui che provoca la narcosi nel protagonista, quella sospensione obbligata dell'azione, quella potente epoché che inibisce la gelosia e i germi della reazione istintiva di fronte alla perdita di possesso della donna amata, di fronte allo scempio del suo corpo che lei stessa è costretta ad ammettere e che farebbe impazzire ogni uomo. Di fatto, l'azione provocherebbe la fine - una fine qualsiasi - dell'avventura di Silvia e di conseguenza l'impossibilità di Filippo (di Parise) di proseguire il suo "studio". Egli percepisce il pericolo in cui si trova Silvia, ma non vuole fermare il suo descensus ad inferos: vuole restare (come Parise, come il lettore) a vedere il seguito di questa storia, senza interromperla, da spettatore. Per questo - ha scritto Garboli - quello di Filippo è "un presentimento oscuramente colpevole, perché direttamente interessato allo sviluppo della realtà divinata, e quindi complice nel determinarne l'esito sciagurato" (p. IX).

Nella struttura concentrica e ripetitiva del romanzo, il nucleo tematico centrale si moltiplica in modo psichedelico: struttura identica, cioè, e variazioni minimali. Di fronte al fantasma dell'ossessione (la moglie che esegue come un rito sinistro la fellatio sul sesso del ragazzo), immaginato, divinato, sognato o raccontato direttamente dalla moglie, Filippo prova innanzitutto la bordata destabilizzante dell'odore del sangue: gelosia, impeto violento, sconvolgimento, paura, invidia della gioventù del ragazzo, terrore della vecchiaia incipiente e dell'impotenza senile. In questi frangenti potrebbe agire d'istinto, intervenire con forza e violenza per interrompere il rapporto di Silvia col ragazzo. Ma sempre, regolarmente, provvidenzialmente, subentra in lui la "narcosi", uno stato di sonnambulismo che anestetizza l'infezione della gelosia, disarma l'istinto a difendere la propria compagna e a battersi per lei: tramite la narcosi, esattamente come attraverso l'anestesia, cioè in un "sonno artificiale e non naturale" (p. 12), Filippo passa dallo stato "animale", potenzialmente attivo e istintivo, ad una passività completamente razionale dove anche solo l'ipotesi dell'azione è negata. Manuela Brunetta ha colto nelle apparizioni seriali della narcosi il "freudiano "principio della coazione a ripetere"”. ${ }^{23}$ Pare

23. Brunetta M., Lutto, op. cit., p. 66. 
che sia Filippo infatti a cercare inconsciamente quello stato che gli permette di astrarsi dalla realtà e dalla preoccupazione per la sorte della moglie, che gli garantisce il principio di non-azione come premessa necessaria alla riflessione e al ragionamento. La narcosi di Filippo non è diversa dall'ebbrezza che si cerca, temendola ma allo stesso tempo accarezzandola, in una droga. Gli serve perché è la garanzia dell'immutabilità:

Ma ecco, a questo punto dei miei pensieri, scendere su di me quella narcosi, quell'anestesia benefica che serviva a lasciare le cose come stavano. (p. 218; nostro corsivo)

Gli consente cioè di superare l'infezione sentimentale per restare da solo ad osservare e a ragionare. Ciò che veramente interessa a Filippo, e a Parise, è il ragazzo di cui Silvia s'è innamorata, quel ragazzo che appartiene a una nuova razza di giovani dai valori e dai comportamenti indecifrabili. $E$ in effetti l'inconsueta condotta di Filippo di fronte al tradimento delinea e negativo un'altra faccia che potremmo definire la "tentazione giornalistica” di Parise: l'idea di sfruttare il viaggio di Silvia negli abissi della nuova, pericolosa e indecifrabile gioventù per capire, sapere, conoscere il mondo contemporaneo e le sue mutazioni. ${ }^{24}$ Accantonare la gelosia, l'istinto possessivo e l'apprensione del marito comune per guardare con occhio distaccato e critico. L'unico modo, insomma, per giungere alla conoscenza diretta con la freddezza necessaria, all'indagine scientifica. Il professionista (l'analista) prende il sopravvento in Filippo sul marito sofferente, ${ }^{25}$ con un espediente narrativo che nasconde la vera ratio sottesa al romanzo: la tentazione istintiva del reporter, la sete di conoscenza di uno straordinario giornalista come Goffredo Parise. Che infatti, in un intervento pronunciato a Napoli il 22 ottobre 1976, presso la Pontificia Facoltà Teologica, si esprimeva così a proposito della nuova cultura consumistica (nata dall'"innesto del pragmatismo consumistico americano nella tradizione materialistica della cultura italiana, popolare e non, di matrice cattolica") e del suo rapporto coi giovani:

24. Su Parise giornalista, $c f r$. Zanzotto A.: "L'interesse di Parise per "tutto ciò che è umano", anche se corroso dai più crudi dubbi sul senso generale dell'uomo e del suo cammino contorto e vagolante, non viene mai meno. Egli, pur conservando un suo distacco, una sua imprendibilità, vuole essere sempre "sul campo", testimone che mai si perdonerebbe d'essere stato assente là dov'è in gioco una possibilità di capire, amare, partecipare, stupirsi, ribellarsi, sdegnarsi, di fronte agli eventi, all'immenso teatro della realtà" (Scritti sulla letteratura, a cura di G. M. Villalta, vol. II, Aure e disincanti nel Novecento letterario, Milano, Mondadori, 2001, p. 273).

25. "La curiosità, professionale personale e passionale, mi pungolava e sapevo anche come soddisfarla, senza dare troppo nell'occhio" (p. 45). 
È un fenomeno al tempo stesso ripugnante e attraente. Ripugnante alle categorie, a tutte le categorie della vecchia cultura (in cui comprendo cultura cattolica, cultura liberale e la brevissima cultura marxista), ma attraente per qualche cosa che non appartiene a nessuna categoria culturale e che io chiamo "vitalità". Non c'è alcun dubbio, positiva o negativa che sia o la si voglia giudicare, la nuova cultura possiede una enorme vitalità. Se tale vitalità sia simile alla vitalità dei fenomeni degenerativi e necrotici dei tessuti (il tessuto sociale del nostro paese in questo caso) non sta a me giudicare perché, per farlo, dovrei afferrarmi saldamente a qualche pilastro della vecchia cultura, come per esempio la morale cattolica, il razionalismo laico o la dialettica marxista. Afferrarmi saldamente a uno di questi pilastri per me non è possibile perché l'attrazione verso la vitalità, di qualunque specie essa sia, è più forte, in me, di qualunque pilastro interpretativo e metodologico. Preferisco il nulla, il vacuum, che mi può dare le vertigini ma allo stesso tempo mi proietta nella conoscenza diretta delle cose. $^{26}$

Parole illuminanti se lette in funzione del romanzo. Quelle "vertigini" di cui Parise parlò a Napoli non corrispondono forse con lo stato di narcosi che cala sul protagonista nel suo disperato tentativo di raggiungere la verità? Di fatto, quelle vertigini e quella narcosi altro non sono che nomi diversi di una stessa vocazione esistenziale. Di fronte al mutamento epocale dei costumi, di fronte alla "nuova razza" dal ghigno nevrotico e assurdo che minaccia l'Italia, Pasolini si ribella, reagisce attivamente e violentemente con parole e azioni. Parise invece, attraverso la narcosi e le vertigini, trova una reazione "implosiva": sprofonda nella prospettiva dello sguardo contemplativo che si lascia inghiottire passivamente dal flusso magmatico della realtà. ${ }^{27}$

26. Rielaborazione scritta pubblicata col titolo Nuovo potere e nuova cultura in "Rassegna di teologia", n. 6, XVII, novembre-dicembre 1976. Ora in Parise G., Opere, a cura di B. Callegner e M. Portello, vol. II, Milano, Mondadori, 1989, p. 1403.

27. Secondo Zanzotto il Parise giornalista, pure avvicinabile a Pasolini per "una forte volontà critica e pedagogica", se ne distingue tuttavia perché "non redarguisce, non propone, si dichiara quasi perdente in partenza, finisce per constatarsi egli pure italiano con tutti i difetti degli italiani, fatto della loro stessa "materia", dello stesso humus. L'indignazione rimane come bloccata da un'indulgenza sofferente e scarsamente convinta, e finisce quasi sempre nel gusto amaro di una quasi-connivenza" (Aure e disincanti nel Novecento letterario, op. cit., p. 275). 